\title{
ANALYSIS OF CUSTOMER SENTIMENT ON PRODUCT FEATURES AFTER THE OUTBREAK OF CORONAVIRUS DISEASE (COVID-19) BASED ON ONLINE REVIEWS
}

\author{
Kim, Jinju; \\ Park, Seyoung; \\ Kim, Harrison \\ University of Illinois at Urbana-Champaign
}

\begin{abstract}
The outbreak of the coronavirus disease not only caused many deaths worldwide but also severely affected the development of the global economy, such as supply chain disruptions, plummeted demand, unemployment, etc. These social changes have led to changes in customers' purchasing patterns. Therefore, it is more important than ever for manufacturers to quickly identify and respond to changing customer purchasing patterns and requirements. However, few studies have been done on dynamic changes in customer preferences for product features following COVID-19 spread. This study aims to investigate the dynamic change of customer sentiment on product features following COVID-19 through sentiment analysis based on online reviews. The proposed methodology consists of two main processes: feature extraction and sentiment analysis. After finding a specific feature of the product through feature extraction, the words used to mention the feature in the review were analyzed for sentiment analysis of customers. To demonstrate the methodology, a case study is conducted using new and refurbished smartphone reviews to investigate the dynamic changes in customer sentiment during COVID-19.
\end{abstract}

Keywords: Semantic data processing, Machine learning, Market implications

\section{Contact:}

Kim, Harrison

University of Illinois at Urbana-Champaign

Industrial and Enterprise Systems Engineering

United States of America

hmkim@uiuc.edu

Cite this article: Kim, J., Park, S., Kim, H. (2021) 'Analysis of Customer Sentiment on Product Features after the Outbreak of Coronavirus Disease (COVID-19) based on Online Reviews', in Proceedings of the International Conference on Engineering Design (ICED21), Gothenburg, Sweden, 16-20 August 2021. DOI:10.1017/pds.2021.46 


\section{INTRODUCTION}

The outbreak of coronavirus, which is currently termed as the coronavirus disease 2019 (COVID-19), has been a direct threat to human beings and leads to a global crisis. As the virus spreads rapidly from person to person, the number of confirmed patients and deaths is exploding around the world. Nevertheless, because there is no vaccine or antivirals agent approved for prevention or treatment yet, only methods to slow the spread of the virus such as social distancing or shutdown (i.e., limiting close faceto-face contact with others) are being used. The implementation of policies and practices for social distancing has brought a variety of changes not only to individuals' lives but also in our society, including governments, industrial companies, and academic institutions (Sarkis et al., 2020). In particular, they have had severe impacts on global economic developments such as supply chain disruptions, shutdowns, plummeted demand, and unemployment (Aslam et al., 2020).

Given these policy changes and the global economic downturn, it has been expected that customers' product buying patterns change: Surge in e-commerce, preference for trusted products (i.e., they prefer products with less troublesome for return processes and use), reduced spending on non-essential goods, and shock to loyalty due to changes in the major drivers for consumers to choose brands (i.e., when consumers can't find their preferred product from their preferred brand, there is an increased tendency for many consumers to try different brands) (Kohli et al., 2020). These changes in customer buying patterns due to the emergence of COVID-19 has led product manufacturers to face uncertainty and challenges. To attract new customers while maintaining the loyalty of existing customers in this crisis, it is more important than ever to capture the different needs of consumers and identify the key motivations for consumers to choose products or brands. However, to the best of our knowledge, few studies have been done on dynamic changes in customer preferences (sentiments) for product features following a major crisis such as COVID-19 spread.

To tackle this issue, this study aims to investigate the dynamic change of customer sentiment on product characteristics following COVID-19 through sentiment analysis. Sentiment analysis is one of the main fields of natural language processing (NLP) and aims to determine the attitude of a person concerning some topic or the overall contextual sentiment polarity of a document (Suryadi \& Kim, 2018; Turney \& Littman, 2003). Given that online purchases have increased during COVID-19 (Krakowski, 2020) and quick analysis and response in this crisis are needed, analysis of online reviews during COVID-19 has an advantage of being able to detect dynamic changes in customers' preferences for a short period compared to traditional methods such as surveys and interviews. This paper presents empirical research on dynamic changes in customer responses for product features caused by the spread of COVID-19 based on online reviews. The following two research questions are addressed in this paper:

1. Will customers during COVID-19 have the same or similar sentiments as before COVID-19 toward the features of new and refurbished products?

2. If the customer's sentiments for the feature had changed before and during COVID-19, how would the customer's sentiments for each feature of the new and refurbished product be different?

In order to find the answers to these research questions, this study aims to find out important features of a specific product and analyze customer reviews for the features to determine whether there is a significant change in customer sentiment before and during COVID-19. Specifically, this study analyzes the ratio of reviews for specific features to the total review, the ratio of positive or negative reviews, the customer's sentiment score, and the adjectives that determine the sentiment score. In addition, statistical verification was performed to determine the significance of the difference between the results before and during COVID-19. The rest of the paper is organized as follows. Section 2 reviews relevant prior work/literature on this topic. Section 3 presents a description of the application process and methodology of customer sentiment analysis through a smartphone example. Section 4 illustrates the analysis results derived through the analysis process. Finally, conclusions and future research are discussed in section 5 .

\section{LITERATURE REVIEW}

\subsection{Research on the impact of COVID-19 on consumer consumption}

After the pandemic was declared, most countries implemented social distancing policies to prevent the spread of the virus. As a result, stores, businesses, and schools were closed, and the working mode 
changed to telecommuting (Sarkis et al., 2020; Aslam et al., 2020). These social and economic changes have led consumers to cancel, limit, or redirect spending, and demand has changed dramatically (Dunn et al., 2020). Several studies investigated consumer changes due to COVID-19 through traditional methodologies such as questionnaires and interviews. Bhargava et al. (2020) surveyed whether consumer consumption declined during COVID-19 for each retail category. As a result, although consumption of groceries and household supplies increased, other items (e.g., electronics, automobiles, gasoline, clothing, etc.) have decreased significantly, and consumers also answered that they would use less than usual for these items. The COVID-19 pandemic has affected the demand for new products as well as remanufactured or refurbished products. Through a report by re-commerce platform Cashify, Koshi (2020) revealed that the demand for refurbished smartphones has increased due to COVID-19 pandemic. This survey indicated that price and affordability become an important criterion for purchasing refurbished smartphones, indicating that they have become cautious about consumption during COVID-19. In addition, some studies were conducted to investigate the impact of COVID-19 on consumers through real data. Alexander \& Karger (2020) and Dunn et al. (2020) used the daily data sources such as cell phone data and card transaction data to investigate the impact of stay-at-home orders due to COVID19 on customer spending. Their estimates showed a decrease in spending in March after stay-at-home orders and provided early point estimates for that decline across several categories. Most of the studies mentioned above only provided insights that customer consumption changed due to COVID-19, and did not address the issue of how to apply these results to product design or production processes.

\subsection{Analysis of online reviews for product design}

Recently, research on the methodology using online reviews for product design improvement and development is actively being conducted. There are two main types of research on online analysis for product design. One is to extract product features that the customer considers important from customer reviews and the other is to analyze sentiment indexes such as adjectives related to feature words or the rating of reviews containing feature words.

Zhou et al. (2020) adopted latent Dirichlet allocation (LDA) for analyzing the review data. LDA returned the topic mentioned in reviews and those that are related to product features were selected. Tuarob \& Tucker (2015) used the rule-based method for feature extraction. With seed features and pre-defined rules, they analyzed the comments in Twitter. Then, the rules detected new features not mentioned in seed features. Suryadi \& Kim (2018) used word embedding and clustering. Words in online review sentences were embedded into vector space and then grouped into clusters by X-means clustering. The clusters with the center word that appears in product manuals were selected as feature clusters. Park \& Kim (2020) extracted phrases from the review data and embedded them using word vectors composing the phrase. Then, spectral clustering was applied to the embedded phrases. The method returned clusters consisting of sub-feature terms.

Another research stream is customer sentiment analysis. Bag et al. (2019) developed a framework to build a prediction model based on the social perception score of the brand and review's polarity. The result provided product features that caught the customer's attention. Suryadi \& Kim (2018) identified the importance of product features on the sales rank by analyzing the relationship between each customer's sentiment score on features and the sales rank of the purchased product.

However, to the best of our knowledge, there are few papers related to the dynamic changes in customers' sentiments about the features of products in connection with massive-scale disruption in the market such as COVID-19. Considering the advantages of online review analysis, which allows obtaining information in a short time compared to the traditional methods of interviews or surveys, online review analysis is expected to help manufacturers in crisis develop and improve their new product and customer marketing strategies.

\section{METHODOLOGY AND APPLICATION}

The purpose of this study is to investigate the dynamic changes in customers' sentiments about the features of products in connection with COVID-19 based on online reviews. Figure 1 illustrates the workflow of the proposed methodology. The proposed methodology consists of two main processes: feature extraction and sentiment analysis. The feature extraction is composed of three steps: (i) preprocessing, (ii) phrase embedding, and (iii) phrase clustering. The methodologies used in this study 


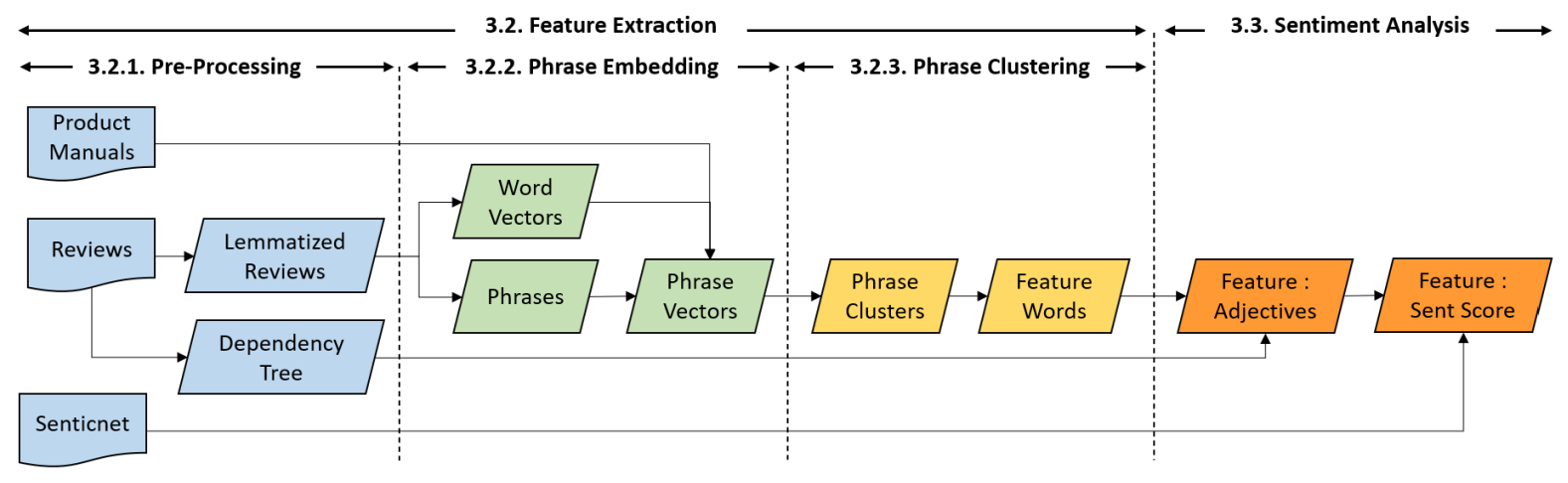

Figure 1. Flowchart of the overall process

are based on previous relevant frameworks and studies focusing on feature extraction and customer sentiment analysis (Suryadi \& Kim, 2018; Park \& Kim, 2020).

To demonstrate the methodology, a case study is conducted using new and refurbished smartphone reviews to investigate the dynamic changes in customer sentiment during COVID-19 through online review analysis. COVID-19 has had a profound impact on the global smartphone market. The smartphone market has experienced the worst decline in history due to the pandemic. During the pandemic, smartphone sales in the first and second quarters in 2020 declined significantly (Vanian, 2020) due to travel restrictions, retail closures, and prudent spending on nonessential products, according to market tracker Gartner. Smartphone manufacturers need to capture customers' changes more sensitively and respond more quickly than ever before. This section provides a step-by-step process of the sentiment analysis for two databases of online smartphone reviews obtained by dividing the period before (October 2019 to February 2020) and during (March to July 2020) COVID-19.

\subsection{Data collection}

In this study, two types of data are collected: (i) online customer reviews; (ii) product manual documents that are distributed by manufacturers. The online smartphone review data, including new and refurbished products, was collected through Amazon.com. Among the smartphones included in the Amazon 100 list as of July 11, 2020, phones that were sold simultaneously during the analysis period (before- and during COVID-19) were targeted. The total number of reviews is 15,811 written from October 20, 2019, to July 11,2020 . Among them, the number of new smartphones and refurbished smartphones reviews is 5,856 and 9,955, respectively. The total number of analyzed smartphone models is 42 , of which the number of new products is 25 and the number of refurbished products is 17 . For the authenticity of the data, only verified reviews provided by Amazon were used.

Also, the product manuals used in this study are documents distributed by manufacturers, all of which are provided online. Specifically, manual documents for seven different smartphones were used. Although this is a smaller number than the number of smartphones covered in this study, it is assumed that the functions and features of smartphones are similar, so that it will be sufficient to cover the representative features of smartphones by analyzing the smartphone manuals of these seven different brands and models.

Table 1 contains the details of the collected data. As shown in Table 1, the collected reviews are divided into four groups by two criteria: one group is about the product category and is divided into new products and refurbished products accordingly. The other is about the timing and is divided into before and during COVID-19. More than 100 countries around the world have introduced full or partial closures by March

Table 1. Details of the collected data

\begin{tabular}{clcc}
\hline \hline & & Pre COVID-19 & During COVID-19 \\
& & $(10 / 20 / 2019 \sim 02 / 29 / 2020)$ & $(03 / 01 / 2020 \sim 07 / 11 / 2020)$ \\
\hline \multirow{3}{*}{ Number of reviews } & New & 3,543 & 2,313 \\
& Refurbished & 5,005 & 4,950 \\
\cline { 2 - 4 } & Total & 8,548 & 7,263 \\
\hline \hline
\end{tabular}


2020, affecting billions of people since the World Health Organization (WHO) declared it an epidemic on March 11 (Dunford et al., 2020). Therefore, in this study, the baseline for before and during COVID19 was set to March based on the time when closure was made in most countries including the United States.

\subsection{Smartphone feature extraction}

For smartphone feature extraction, the methodology suggested by Park \& Kim (2020) was modified and used in this research. As shown in Figure 1, feature extraction is composed of three main processes: pre-processing, phrase embedding, and phrase clustering.

\subsubsection{Pre-processing review data}

In this study, Spacy library in PYTHON was used for pre-processing the review data. The collected reviews have been organized by removing punctuation marks and changing uppercase letters to lowercase letters. Then, all words were lemmatized. Stopwords that refer to the English words which does not add much meaning to a sentence were not removed because it would affect the phrase extraction step to be performed in the next section. Spacy nlp analyzes the sentences and returns POS-tagging of each word and a dependency tree which represents the linguistic structure of a sentence.

\subsubsection{Phrase Embedding}

First, words in review data were embedded into vectors. Gensim library in PYTHON was used for word embedding. The lemmatized words from the previous stage were used for an input to word2vec modeling. The parameters were set as follows: the dimension of the vector is 100 , the number of windows is 2 , and the minimum word count is 8 . After training, Gensim returned a set of word vectors. Park \& Kim (2020) filtered word vectors using POS-tagging to collect noun vectors only. However, this research skips the filtering process to extract more diverse feature-related phrases.

Next, phrases in review data were extracted by using the Noun_chunk method and Textrank method of Spacy library in PYTHON. For the simplicity of the process, phrases consisting of two words were considered in this research. To obtain feature-related terms only, the phrases containing a word that are not mentioned in product manual documents were removed. The remaining phrases were embedded by Eq. 1 from Park \& Kim (2020) where $\vec{W}_{1}$ and $\vec{W}_{2}$ represent the vector of the first and second word in the phrase respectively. The phrase vector is constructed by a weighted sum of these two word vectors. The weight for each word, $a_{i}$, is the ratio of frequencies in manual documents. To be specific, $a_{i}$ is calculated by dividing the frequency of word $i$ by the total frequency of two words.

$$
\begin{gathered}
\text { Phrase Vector }=a_{1} \vec{W}_{1}+a_{2} \vec{W}_{2} \\
a_{1}=\frac{\operatorname{Freq}\left(W_{1}\right)}{\operatorname{Freq}\left(W_{1}\right)+\operatorname{Freq}\left(W_{2}\right)} \\
a_{2}=\frac{\operatorname{Freq}\left(W_{2}\right)}{\operatorname{Freq}\left(W_{1}\right)+\operatorname{Freq}\left(W_{2}\right)}
\end{gathered}
$$

\subsubsection{Phrase clustering}

To group similar phrases, spectral clustering was applied to the embedded phrases. The number of clusters was chosen by conducting multiple trials with $K=48$ to 55 . In this case study, $K=54$ was chosen by manually analyzing the items in resulting clusters. Among 54 clusters, 8 feature-related clusters were selected and they were summarized into 6 feature categories as shown in Table 2. The first column is the subjective feature labeling based on cluster members and the second column contains related phrases.

\subsection{Sentiment analysis}

In general, sentiment analysis is conducted by defining target words and collecting sentiment indexes related to those words. In this study, target words are feature-related words and they were obtained by decomposing feature-related phrases from the previous section. These target words include 'screen', 'resolution', 'memory', 'selfie, etc. And then, various indexes have been used for detecting sentiments: (i) the sentiment score of the adjective connected to the target word; (ii) the sentiment level of the 
Table 2. Extracted feature phrases

\begin{tabular}{cl}
\hline \hline Cluster label & Extracted phrases \\
\hline Screen & screen size, large screen, screen resolution, screen technology, screen brightness, secreen ratio, etc. \\
\hline Memory & more storage, gb memory, enough space, internal memory, gb ram, more memory, extra storage, etc. \\
\hline Camera & front camera, selfie camera, rear camera, main camera, pixel camera, camera lens \\
\hline Battery & battery capacity, mah battery, huge battery, large battery, big battery, small battery, battery life, etc. \\
\hline Security & fingerprint reader, fingerprint sensor, fingerprint unlock, finger scanner, face recognition, etc. \\
\hline Price & price drop, great price, great value, decent price, affordable price, more money, bargain price, etc. \\
\hline \hline
\end{tabular}

sentences containing the target word; (iii) the average rating of the reviews mentioning the target word. This study follows the methodology of Suryadi \& Kim (2018) using the first approach.

First, each review sentence was scanned to check whether the sentence contains feature words. If it does, the sentence was further analyzed to find the adjectives connected to the feature word. As shown in Figure 1, the dependency tree from Section 3.2.1 was used in this task. All the adjectives that are directly or indirectly connected to the feature word were discovered. The analysis result created the feature word and sentiment pairs for every sentence.

Next, based on these pairs, the reviewer's sentiment score for each feature was computed. All the adjectives in the pairs were quantified by Senticnet, the dictionary of sentiment scores. To be specific, a detected adjective became an input for Senticnet and then, Senticnet returned the polarity (positive/negative) and the magnitude of the sentiment for the given adjective. For example, Senticnet returned 0.857 for 'great' and -0.27 for 'bad'. And then, the mean value of all scores became the reviewer's sentiment for the target feature. If the reviewer does not mention a specific feature, the sentiment score was set to zero. Also, when the reviewer mentions a particular feature word, but there are no adjectives connected to that word, the sentiment score for that feature was also recorded as zero.

\section{RESULTS AND DISCUSSION}

This section presents the results of customer sentiment analysis based on online reviews. The subsection is divided into new and refurbished products. It shows the change in the number of positive and negative reviews and the change in the customer's sentiment score for each feature that appears before and during COVID-19.

\subsection{Comparison of feature mention rates in reviews before and during COVID-19}

In this subsection, the ratio of the number of reviews mentioning product features to the total number of reviews was calculated, which is termed the degree of customer attention in this paper. The change in the number of customer comments on a product feature mainly indicates a change in the customer's current state of attention and the importance of the feature in the product (Zhang et al., 2018). In addition, the degree of satisfaction and dissatisfaction is the percentage of positive and negative comments about each feature out of the reviews that mention certain features, respectively. Through this analysis, it is possible to predict whether there is a change in features that customers consider crucial before and during COVID-19 and whether the change in customer attention is positive or negative.

\subsubsection{New products}

In the case of new products, the total number of reviews decreased from 3,543 to 2,313 , about $35 \%$ reduction during the pandemic (See Table 1). Considering the correlation between sales volume and number of reviews, it can be predicted that sales volume of new products has decreased. Table 3 shows the changes in the degree of attention, satisfaction, and dissatisfaction by feature with new products before and during COVID-19. For the degree of attention, although the number of reviews decreased during COVID-19, mention of all features increased. These results show that although sales of new products since COVID-19 have dropped significantly, customers who purchase at this time are paying attention to features and are willing to purchase them carefully. The levels of satisfaction and dissatisfaction are shown in columns 4 through 5 of Table 3 . Regarding the degree of satisfaction, positive opinions decreased during COVID-19 in memory, camera, battery, and price, excluding screen and security. In 
Table 3. Customer feature mention rate (New products)

\begin{tabular}{|c|c|c|c|c|}
\hline New & Product features & Degree of attention & Degree of satisfaction & Degree of dissatisfaction \\
\hline \multirow{6}{*}{$\begin{array}{l}\text { Before } \\
\text { COVID-19 }\end{array}$} & Screen & $20 \%$ & $51 \%$ & $18 \%$ \\
\hline & Memory & $5 \%$ & $56 \%$ & $12 \%$ \\
\hline & Camera & $28 \%$ & $61 \%$ & $10 \%$ \\
\hline & Battery* & $30 \%$ & $45 \%$ & $24 \%$ \\
\hline & Security & $31 \%$ & $21 \%$ & $9 \%$ \\
\hline & Price* & $26 \%$ & $62 \%$ & $9 \%$ \\
\hline \multirow{6}{*}{$\begin{array}{l}\text { During } \\
\text { COVID-19 }\end{array}$} & Screen & $22 \%$ & $52 \%$ & $19 \%$ \\
\hline & Memory & $6 \%$ & $52 \%$ & $12 \%$ \\
\hline & Camera & $30 \%$ & $57 \%$ & $10 \%$ \\
\hline & Battery* & $35 \%$ & $40 \%$ & $28 \%$ \\
\hline & Security & $38 \%$ & $22 \%$ & $11 \%$ \\
\hline & Price* & $27 \%$ & $58 \%$ & $12 \%$ \\
\hline
\end{tabular}

* The result is statistically significant at the $\alpha=0.05$ level

terms of dissatisfaction, the results show that memory and camera remained unchanged, and negative comments increased on the screen, battery, security, and price.

A chi-square test was performed to find the significance of the difference between the number of positive and negative reviews before and during COVID-19. Through this test, it is possible to check whether there is a significant difference in the number of positive and negative reviews for a specific feature. As a result of the chi-square test, the change in the number of positive/negative comments to the battery and price of the new product before and during COVID-19 showed a significant difference, with a significance level of less than 0.05 . Based on these results, it can be seen that the battery and price have statistically significant decrease in positive reviews and increase in negative reviews before and during COVID-19.

\subsubsection{Refurbished products}

On the other hand, in the case of refurbished products, the total number of reviews decreased slightly from 5,005 to 4,950 during the pandemic but did not drop as much as with new products (See Table 1). Table 4 shows the changes in the degree of attention, satisfaction, and dissatisfaction by feature with refurbished products before and during COVID-19. The results show that customer attention has increased in all other features except memory. There was no change in customer attention in the memory feature before and during COVID-19. For the degree of satisfaction, positive opinions on screen, battery and security increased except for memory and cameras during COVID-19. For the degree of dissatisfaction, there was no change in battery, security, and price, and negative comments about the screen and memory are decreased. However, the results of the chi-square test showed that there was no significant difference before and during COVID-19 for all features of the refurbished products.

\subsection{Comparison of customer sentiment score before and during COVID-19}

In this study, not only the number of reviews mentioning the product features but also the reviewer's sentiment score for each feature was calculated and analyzed. This analysis targets the reviews mentioned

Table 4. Customer feature mention rate (Refurbished products)

\begin{tabular}{|c|c|c|c|c|}
\hline Refurbished & Product features & Degree of attention & Degree of satisfaction & Degree of dissatisfaction \\
\hline \multirow{6}{*}{$\begin{array}{l}\text { Before } \\
\text { COVID-19 }\end{array}$} & Screen & $14 \%$ & $35 \%$ & $21 \%$ \\
\hline & Memory & $3 \%$ & $52 \%$ & $12 \%$ \\
\hline & Camera & $12 \%$ & $46 \%$ & $10 \%$ \\
\hline & Battery & $30 \%$ & $48 \%$ & $20 \%$ \\
\hline & Security & $26 \%$ & $16 \%$ & $7 \%$ \\
\hline & Price & $27 \%$ & $68 \%$ & $9 \%$ \\
\hline \multirow{6}{*}{$\begin{array}{l}\text { During } \\
\text { COVID-19 }\end{array}$} & Screen & $17 \%$ & $38 \%$ & $20 \%$ \\
\hline & Memory & $3 \%$ & $46 \%$ & $17 \%$ \\
\hline & Camera & $14 \%$ & $45 \%$ & $12 \%$ \\
\hline & Battery & $36 \%$ & $50 \%$ & $20 \%$ \\
\hline & Security & $31 \%$ & $18 \%$ & $7 \%$ \\
\hline & Price & $33 \%$ & $68 \%$ & $9 \%$ \\
\hline
\end{tabular}




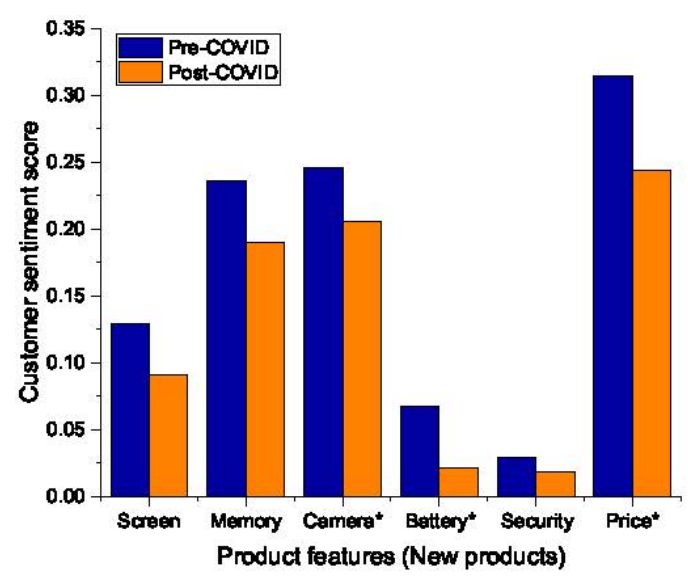

(a)

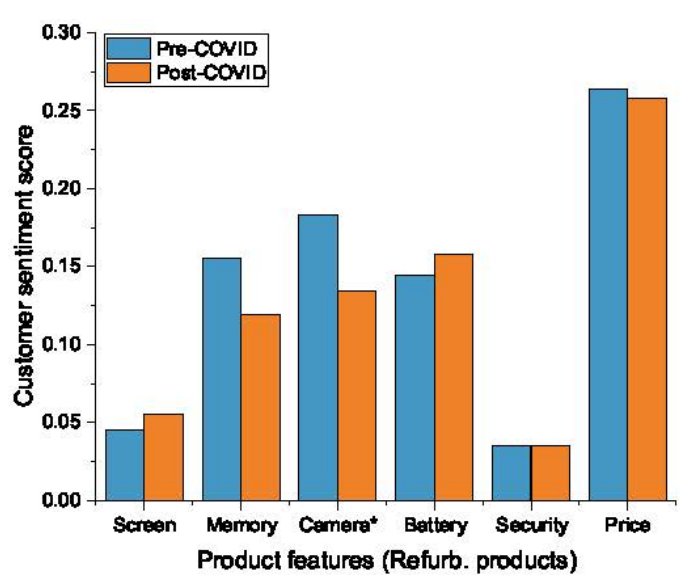

(b)

* The result is statistically significant at the $\alpha=0.05$ level

Figure 2. (a) The difference in customer sentiment score (New products), (b) The difference in customer sentiment score (Refurbished products)

for each feature and scores the sentiments of the customers who wrote the reviews. The customer's sentiment score ranges from -1 to 1 , where -1 is very negative, 0 is neutral, and 1 is very positive. The average sentiment score for each feature shows how customers feel about product features at each time period, and whether there is a difference between the two periods. Figure 2 shows the difference in average customer sentiment scores before and during COVID-19 for new and refurbished products.

For new products, the results show that the customer sentiment score was lowered in all features during COVID-19, as shown in Figure 2 (a). A two-sample $t$-test was performed to assess whether the average sentiment score reduction for each feature due to COVID-19 was significant. As a result of statistical verification, there were significant differences in the camera, battery, and price for new products. These results show that customer satisfaction with new products has decreased significantly for the camera, battery and price during COVID-19.

Figure 2 (b) represents the difference in average customer sentiment scores before and during COVID19 for refurbished products. In the case of refurbished products, the average sentiment score of the screen and battery increased, while memory and camera decreased. Refurbished products also confirmed the significance of the sentiment score difference through $t$-test, and the result showed that customer satisfaction with the camera was significantly reduced.

\subsection{Analysis of adjectives that determine sentiments}

According to previous analysis results (Section 4.2), there were significant changes in customer sentiment about specific features of the new and refurbished products before and during COVID-19. The results indicated that for new products, cameras, battery, and price factors negatively affect customer sentiment, whereas, for refurbished products, cameras negatively affect customer sentiment. In this subsection, the top 20 adjectives used in each feature of the review were analyzed to see which factors contributed to this dissatisfaction. Among the top 20 adjectives used before and during COVID-19, adjectives used at the same time in two periods were classified as common adjectives, and adjectives that were not used were classified as unique adjectives. For common adjectives, the adjective usage ratio - frequency of use of adjectives divided by the number of feature mentions in the review - was used to examine the change in the frequency of adjective use before and during COVID-19. The unique adjectives attempted to identify new issues that appeared during COVID-19 by categorizing adjectives newly appearing in the top 20 adjectives ranking during COVID-19. Through this analysis, it is possible to find the factors of satisfaction/dissatisfaction with the feature of the product during COVID-19 and use it for future design improvement.

\subsubsection{New products}

The top 20 adjectives of new products that determine customer sentiment were extracted and analyzed for camera, battery, and price. The top 20 adjectives in the camera were largely classified as those 
related to overall evaluation, photography, and use. The adjective usage ratio in the overall evaluation of the camera confirmed that the use of adjectives, which represent most positive assessments, decreased during COVID-19 ('good'; 'amazing'; 'awesome'; 'wonderful'). In particular, during COVID-19, the use of adjectives related to photography which is the detailed features of the camera increased compared to before COVID-19 ('many'; 'nice'; 'front'; 'clear'). In the case of unique adjectives, additional issues with the rear camera and the sound from the camera when taking pictures were mentioned during COVID-19 ('rear'; 'sound'). These results show that customers' positive overall evaluation of the camera during COVID-19 has decreased, and customers are more interested in the detailed functions of the front and/or rear camera than before COVID-19.

For the common adjective usage of the battery, it was confirmed that the percentage of positive adjectives used in the overall evaluation decreased compared to before COVID-19 ('good'; 'great'; 'amazing'; 'well'). For battery capacity, the use of positive adjectives decreased ('big'; 'huge') and the use of negative adjectives increased ('low'). For charging speed, the use of positive adjectives decreased ('fast'; 'full'; 'quick'). These results confirmed that the battery also decreased between positive adjectives for the overall evaluation and that customers' use of negative adjectives for battery capacity and charging speed increased.

For the price, among the common adjectives, most adjectives that are positive for the price have declined overall since COVID-19 ('great'; 'good'; 'perfect'; 'worth'; 'amazing'; 'affordable'; 'reasonable'; 'nice'; 'incredible'). For unique adjectives, the use of negative adjectives appeared during COVID-19 ('bad'; 'less'). Compared to before COVID-19, the results indicated that the number of customers who positively rated the price of the same product has decreased.

Through the analysis of the set of adjectives that determine the sentiment score of each feature, it was possible to grasp the change in the customer's evaluation and attention level for the detailed features of the new product. For new products, customers during COVID-19 are more likely to pay attention to the detailed specifications of the front and rear cameras, sound problems during taking pictures, battery capacity and charging speed, and price burdens compared to those before COVID-19.

\subsubsection{Refurbished products}

For refurbished products, the top 20 adjectives used to mention the camera in customer reviews were extracted and analyzed. For the overall evaluation of the camera, some adjectives that are positive for the camera have declined since COVID-19 ('good'; 'great'). In addition, the percentage of customers who specifically mentioned the front and rear cameras was higher during COVID-19 ('rear'; 'front'; 'back'). For unique adjectives, the word 'dead' appeared in the top 20 adjective list during COVID-19. According to these results, for refurbished products, customers during COVID-19 are more likely to pay more attention to the detailed specifications of the front and/or rear cameras compared to before COVID-19. In addition, quality checks will be required since camera malfunctions are mentioned in unique adjectives.

\section{CONCLUSION}

This paper analyzes customers' sentiments about various features of smartphones before and during COVID-19 by using online review data. The customer online review data was collected from Amazon and product features were extracted from the review sentences. Then, the collected data was analyzed by categorizing it into product categories (new and refurbished phones) and before/during COVID-19. Based on the classified review data, the customer's sentiment analysis was conducted by analyzing the ratio of reviews for specific features to the total review, the ratio of positive or negative reviews, the customer's sentiment score, and the adjectives that determines the sentiment score.

This analysis results showed the changes in customer sentiment following the spread of COVID-19 through online reviews. First, there are significant changes in customer sentiment about specific features of the new and refurbished products before and during COVID-19. According to the results on the number difference of positive and negative reviews between before and during COVID-19, there was a significant difference in battery and price for new products. Also, the analysis of customer sentiment scores revealed that the camera, battery, and price factors negatively affect customer sentiment for new products, while the camera negatively affects customer sentiment for refurbished products. Also, the set of adjectives that determine the sentiment score of each feature was analyzed 
to identify the changes in customer evaluation and attention in specific features of products amid COVID-19 crisis.

The analysis results of this study have several meaningful implications amid COVID-19 crisis. Through online review analysis, it is easier and faster than traditional methods to identify customer preferences or requirements that have changed due to COVID-19. Some of the results of this study were consistent with the content of questionnaires and interviews conducted in previous studies such as decreased sales of new smartphones, increased demand for refurbished products due to price and affordability. Therefore, product manufacturers can identify changes in customer evaluation and attention to specific features of products amid COVID-19 crisis through this analysis.

Identifying customer changes in COVID-19 era and rapidly applying this information to new product design development and/or improvement is a promising field of future research that must be explored to achieve more economic goals in the situation where everything is uncertain. The results of this study can be extended to future studies that build a model that predicts product demand in COVID-19 era (uncertain based situations) on online customer reviews.

\section{REFERENCES}

Alexander, D., \& Karger, E., 2020. Do stay-at-home orders cause people to stay at home? Effects of stay-at-home orders on consumer behavior.

Aslam, F., Awan, T. M., Syed, J. H., Kashif, A., and Parveen, M., 2020. Sentiments and emotions evoked by news headlines of coronavirus disease (COVID-19) outbreak. Humanities and Social Sciences Communications, 7(1), pp.1-9.

Bag, S., Tiwari, M. K., and Chan, F. T., 2019. Predicting the consumer's purchase intention of durable goods: An attribute-level analysis. Journal of Business Research, 94, 408-419.

Bhargava, S. et al., 2020. Survey: US Consumer Sentiment During the Coronavirus Crisis, Mckinsey and Co., April 5, 2020. Retrieved from https://www.mckinsey.com/business-functions/marketing-and-sales/ourinsights/survey-us-consumersentiment-during-the-coronavirus-crisis

Dunford, D., Dale, B., Stylianou, N., Lowther, E., Ahmed, M., and de la Torre Arenas, I., 2020. Coronavirus: The world in lockdown in maps and charts. Retrieved from https://www.bbc.com/news/world-52103747

Dunn, A., Hood, K., and Driessen, A., 2020. Measuring the effects of the COVID-19 pandemic on consumer spending using card transaction data. US Bureau of Economic Analysis Working Paper WP2020-5.

Kohli, S., Timelin, B., Fabius, V., and Veranen, S. M., 2020. How COVID-19 is changing consumer behavior-now and forever. Retrieved from https://www.mckinsey.com/industries/retail/our-insights/howcovid-19-is-changing-consumer-behavior-now-and-forever

Krakowski, C., 2020. June 2020 Ecommerce Market Trends: Focus on Review Length + Your Questions Answered + Webinar Recording. Retrieved from https:/www.powerreviews.com/blog/june-2020ecommerce-market-trends-focus-on-review-length-your-questions-answered-webinar-recording/

Koshi, L., 2020. Second-hand smartphones see spike in sales amid COVID-19 pandemic, Retrieved from thenewsminute.com/article/second-hand-smartphones-see-spike-sales-amid-covid-19-pandemic-128363

Park, S., and Kim, H., 2020. Improving the accuracy and diversity of feature extraction from online reviews using keyword embedding and two clustering method. Proceeding of ASME 2020 International Design Engineering Technical Conferences and Computers and Information in Engineering Conference

Sarkis, J., Cohen, M. J., Dewick, P., and Schröder, P., 2020. A brave new world: lessons from the COVID-19 pandemic for transitioning to sustainable supply and production. Resources, Conservation, and Recycling.

Suryadi, D., and Kim, H., 2018. A systematic methodology based on word embedding for identifying the relation between online customer reviews and sales rank. Journal of Mechanical Design, 140(12).

Turney, P. D., and Littman, M. L., 2003. Measuring praise and criticism: Inference of semantic orientation from association. ACM Transactions on Information Systems (TOIS), 21(4), pp.315-346.

Tuarob, S., and Tucker, C. S., 2015. Quantifying Product Favorability and Extracting Notable Product Features Using Large Scale Social Media Data. Journal of Mechanical Design, 15.

Vanian, J., 2020. This smartphone maker's sales dropped the most due to COVID-19. Retrieved from https://fortune.com/2020/08/25/apple-samsung-huawei-smartphone-covid-19/

Zhang, H., Rao, H., and Feng, J., 2018. Product innovation based on online review data mining: a case study of Huawei phones. Electronic Commerce Research, 18(1), pp.3-22.

Zhou, F., Ayoub, J., Xu, Q., and Yang, X. J., 2020. A Machine Learning Approach to Customer Needs Analysis for Product Ecosystems. Journal of Mechanical Design, 142. 